RAD Conference Proceedings, vol. 2, pp. 237-242, 2017

www.rad-proceedings.org

\title{
SUPEROXIDE DISMUTASE AND LIPID PEROXIDATION IN CHILDREN AFFECTED BY CELIAC DISEASE
}

\author{
Vesna Stojiljković*, Ljubica Gavrilović, Snežana Pejić, \\ Ana Todorović, Nataša Popović, Ivan Pavlović, Snežana B. Pajović
}

Laboratory of Molecular Biology and Endocrinology, Vinča Institute of Nuclear Sciences, Belgrade, Serbia

\begin{abstract}
Celiac disease (CD) is an autoimmune disorder provoked by the wheat gluten and related proteins from other grains. The only treatment for the patients is a lifelong gluten free diet (GFD). Oxidative stress has been implicated in the pathogenesis of CD. The aim of this study was to examine the modulation of the biochemical response to oxidative stress in children affected by $C D$. The study involved peripheral blood samples and small intestinal biopsies from 69 children diagnosed with CD. According to the histological findings, patients were divided into following groups: Marsh o: normal mucosa with no signs of inflammation $(n=31)$; Marsh 1: mucosa was characterized by intraepithelial lymphocytosis $(n=5)$; Marsh 2: intraepithelial lymphocytosis was accompanied by crypt hyperplasia ( $n=4)$; Marsh 3a: mucosa showed partial villous atrophy $(n=20)$; Marsh $3 b$ : subtotal villous atrophy was present $(n=9)$. For the statistical purposes groups Marsh 1 and Marsh 2 were treated as one (Marsh 1+2, $n=9$ ). The activities and protein levels of copper, zinc superoxide dismutase (CuZnSOD) and manganese SOD (MnSOD), as well as the concentrations of lipid hydroperoxides $(\mathrm{LOOH})$ were determined in intestinal biopsies, while in the peripheral blood, MnSOD activity was not measured, due to the methodological obstacles. CuZnSOD activity in the blood varied significantly between the analyzed groups. Marsh $3 a$ and Marsh $3 b$ had increased CuZnSOD activity comparing to the Marsh $O(P<0.05)$. LOOH concentration also varied significantly. LOOH level was higher in the blood of Marsh $3 a(P<0.001)$ and Marsh $3 b(P<0.05)$, than in Marsh 0. In the biopsy samples, MnSOD activity and $\mathrm{LOOH}$ concentration showed significant differences between the groups, while no significant difference was found for CuZnSOD activity. In comparison to Marsh $O, M n S O D$ activity was significantly elevated in Marsh $3 a(P<0.01)$. Significant increase in LOOH concentration was found in Marsh $3 a(P<0.001)$ and Marsh $3 b(P<0.01)$, comparing to Marsh o. In addition, Marsh 3 a group had higher LOOH concentration than Marsh 1+2. Relative MnSOD and CuZnSOD protein level in the peripheral blood and intestinal mucosa did not vary significantly between the analyzed groups. Positive correlations were found between the severity of mucosal lesion and CuZnSOD activity $(P<0.001)$, as well as $\mathrm{LOOH}$ concentration $(P<0.001)$ in the peripheral blood. Similar correlations were found also in intestinal mucosa: MnSOD: $P<$ o.05; CuZnSOD: $P<0.05$; LOOH: $P<0.001$. Our results show that oxidant/antioxidant balance is disturbed in $C D$ patients with mucosal lesions. An increase in SOD activity as a consequence of oxidant pressure is not enough to maintain the normal level of free radicals, which leads to enhanced lipid peroxidation. These processes persist even in some patients on a long-term GFD.
\end{abstract}

Key words: Celiac disease, Lipid peroxidation, Oxidative stress, Superoxide dismutase

DOI: $10.21175 /$ RadProc.2017.49

\section{INTRODUCTION}

Reactive oxygen species (ROS) are generated as byproducts of metabolic processes in cells. In the physiological conditions the level of ROS is kept in check by an array of antioxidant (AO) enzymes (superoxide dismutase (SOD), catalase (CAT), glutathione peroxidase (GPx), glutathione reductase (GR)), as well as non-enzymatic antioxidants (vitamins $\mathrm{C}, \mathrm{E}, \mathrm{A}$, glutathione). If the production of ROS overwhelms the AO capacity of the cell, oxidative stress occurs. One of the first consequences of the oxidative stress is the oxidation of membrane lipids, a chain reaction known as lipid peroxidation which causes a

\footnotetext{
*vesnas@vin.bg.ac.rs
}

loss of the membrane fluidity, disrupts membrane integrity and functions.

Celiac disease (CD) is an autoimmune disorder provoked by wheat gluten and related proteins from some other grains (barley or rye). In genetically predisposed individuals, gluten activates the immune cascade which leads to the inflammation of intestinal mucosa. The clinical presentation of $\mathrm{CD}$ is extremely heterogeneous [1], ranging from the asymptomatic cases to severe malabsorption of micro- and macronutrients, with a variety of conditions, including diarrhoea, constipation, flatulence, weight loss, vitamin and mineral deficiencies, iron deficiency anaemia and bone disease [2].

CD is one of few disorders for which a specific diet is the only treatment. Persons affected by CD must 
adhere to a lifelong strict gluten-free diet, which usually allows the intestinal mucosa to heal, resolves gastrointestinal symptoms and prevents the complications that may occur if the disease remains untreated.

Previous data $[3,4]$ revealed that oxidative stress and ROS play an important role in the pathogenesis of CD. Our former investigations demonstrated that the antioxidant defense system is notably disturbed in the blood and intestinal mucosa of patients with $\mathrm{CD}[5,6]$. The aim of this study was to investigate alterations in SOD activity and protein levels, as well as the level of lipid peroxidation in blood and intestinal mucosa of pediatric $C D$ patients.

\section{MATERIALS AND METHODS}

\subsection{Subjects}

The study involved peripheral blood samples and small intestinal biopsies from 69 children with celiac disease (32 boys, 37 girls; median age 8 years; range 1.5-16 years) who were attended at the Children's Hospital, Clinical Center of Serbia, Belgrade, Serbia for gastroenterological issues. The Ethical Committee of the Faculty of Medicine, University of Belgrade, approved the study. The blood samples were collected on the day of the intestinal biopsy. In 40 children the diagnosis was made in early childhood and by the time of sampling, they had been subjected to gluten-free diet (GFD) for 2-4 years. The remaining 29 children were diagnosed at the time of the study and were using the gluten containing diet. The diagnosis of celiac disease was based on the revised criteria of the European Society for Paediatric Gastroenterology, Hepatology and Nutrition [7]. Typical villous atrophy was found on the examination of intestinal biopsy specimens in all children on gluten containing diet.

Histological classification was performed according to the modified Oberhuber-Marsh model [8]. Patients were divided into following groups: Marsh o: normal mucosa with no signs of inflammation $(n=31$, all on GFD); Marsh 1: mucosa was characterized by intraepithelial lymphocytosis ( $\mathrm{n}=5$, all on GFD); Marsh 2: intraepithelial lymphocytosis was accompanied by crypt hyperplasia $(n=4$, all on GFD); Marsh 3a: mucosa showed partial villous atrophy $(\mathrm{n}=$ 20, all newly diagnosed and using gluten containing diet); Marsh 3b: subtotal villous atrophy was present ( $\mathrm{n}$ $=9$, all newly diagnosed and using gluten containing diet). For the statistical purposes groups Marsh 1 and Marsh 2 were treated as one (Marsh $1+2, n=9$ ).

\subsection{Sample preparation}

From each patient, 6-8 proximal small intestinal biopsy specimens were obtained. Some of them were used for the histopathological analysis and others were washed in ice-cold saline and frozen at $-70{ }^{\circ} \mathrm{C}$ for SOD and $\mathrm{LOOH}$ assays. Samples were thawed within a week and homogenized in 20 volumes of cold sucrose buffer $\mathrm{pH}$ 7.4. Homogenates were vortexed 3 times for 15 seconds and then kept at $-70{ }^{\circ} \mathrm{C}$. Thawed homogenates were centrifuged (Eppendorf centrifuge 5417R, Eppendorf-Netheler-Hinz GmbH, Hamburg, Germany) at $8600 \mathrm{~g}, 4^{\circ} \mathrm{C}$, for 10 minutes. Supernatants were stored at $-70^{\circ} \mathrm{C}$.

On the day of the biopsy, peripheral blood samples were collected in tubes containing lithium heparin as anticoagulant. The blood was centrifuged at $2500 \mathrm{~g}, 4$ ${ }^{\circ} \mathrm{C}$ for 5 minutes (Eppendorf centrifuge 5417, Eppendorf AG, Hamburg, Germany) to separate the plasma. The plasma was stored at $-70{ }^{\circ} \mathrm{C}$ for the lipid hydroperoxides (LOOH) assay and the buffy coat was discarded. The red blood cells were drawn from the bottom, washed three times in cold saline and haemolysed by adding four packed-cell volumes of icecold demineralised ultra pure water (MilliQ reagent grade water system, Millipore Corp., Bedford, MA, USA). Ethanol-chloroform (5/3 vol/vol) extraction was performed to remove haemoglobin interference. Extracts were stored at $-70^{\circ} \mathrm{C}$ for later analysis.

\subsection{Assays}

All assays were performed spectrophotometrically (Perkin Elmer Spectrophotometer, Lambda 25, Perkin Elmer Instruments, Norwalk, CT, USA).

The SOD specific enzyme activities in blood were expressed as units per gram of haemoglobin (U/g Hb) and in tissue homogenates as units per milligram of protein $(\mathrm{U} / \mathrm{mg})$. Concentrations of $\mathrm{LOOH}$ were expressed in $\mu \mathrm{mol} / \mathrm{L}$.

SOD assay. SOD activity was measured using the Oxis Bioxytech $\AA$ SOD-525TM Assay (Oxis International, Inc., Portland, OR, USA). The kinetic measurement of the change in absorbance at $525 \mathrm{~nm}$ is performed. One SOD-525 activity unit is defined as the activity that doubles the autoxidation rate of the control blank. In blood lysates only CuZnSOD activity was measured, since ethanol-chloroform extraction reagent, necessary for removing the haemoglobin interference, inactivates MnSOD. In tissue homogenates, the total SOD activity is determined as described above. Then, CuZnSOD activity was measured in the same manner, after pretreating samples with ethanol-chloroform reagent. MnSOD activity was then calculated by subtracting CuZnSOD activity from the total SOD activity.

LOOH assay. The concentration of $\mathrm{LOOH}$ was determined by the Oxis Bioxytech $囚 \mathrm{LPO} 560^{\mathrm{TM}}$ Assay (Oxis International, Inc., Portland, OR, USA). The assay is based on the oxidation of ferrous to ferric ions by $\mathrm{LOOH}$ from the sample under acidic conditions. The absorbance at $560 \mathrm{~nm}$ is directly proportional to the LOOH concentration.

Western blotting. Equal amounts of the protein were dissolved in SDS-PAGE sample loading buffer and electrophoresed in polyacrylamide gel (10\%), according to Laemmli (1970). For Western blotting, the proteins were transferred to nitrocellulose membranes. Nonspecific binding sites on the membranes were blocked with TBST (10 mM Tris, $150 \mathrm{mMNaCl}, 0.1 \%$ Tween 20) containing 1\% BSA and then probed with rabbit antiCuZnSOD polyclonal antibody (SOD-100, Stressgen Biotechnologies; 1:7500). After the blots were washed, alkaline phosphatase-conjugated goat anti-rabbit IgG (SAB-301, Stressgen Biotechnologies; 1:7500) was added. Each blot was triplicated and scanned. The density of bands was determined by ImageJ processing software and normalized to the loading control. 


\subsection{Statistical analysis}

The differences between the groups were tested using the analysis of variance (ANOVA) in the blood samples and the Kruskal-Wallis test in tissue homogenates, whereas the significances were evaluated by the Tukey or the Mann-Whitney test in the blood and tissue homogenates, respectively. The Spearman rank-order correlation coefficient was used to investigate the associations between the analyzed parameters and histological lesions. Two-tailed $p$ values are given throughout.

\section{RESULTS}
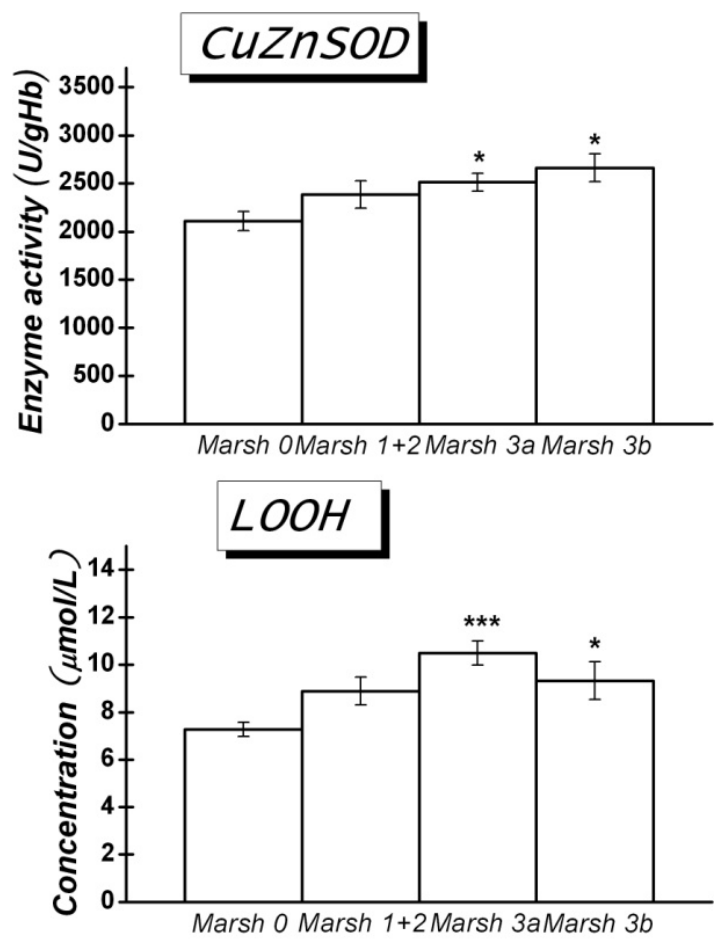

Figure 1. CuZnSOD activity (U/gHb) and LOOH concentration $(\mu \mathrm{mol} / \mathrm{L})$ in peripheral blood of celiac disease patients. ${ }^{* * *} \mathrm{P}<0.001,{ }^{*} \mathrm{P}<0.05$, significantly different from Marsh o group.

The enzyme activity of CuZnSOD and $\mathrm{LOOH}$ concentration in the peripheral blood are shown in Fig. 1. CuZnSOD activity varied significantly between the analyzed groups $\left(\mathrm{F}_{3,65}=4.51, P<0.01\right)$. Both groups with villous atrophy had increased CuZnSOD activity comparing to the Marsh o group $(P<0.05)$. $\mathrm{LOOH}$ concentration also varied significantly between the analyzed groups $\left(\mathrm{F}_{3,65}=11.43, P<0.001\right)$. In the blood of patients with partial and subtotal villous atrophy LOOH level was $~ 45 \%(P<0.001)$ and $\sim 30 \%$ higher $(P<0.05)$ respectively, than in patients with normal mucosa.
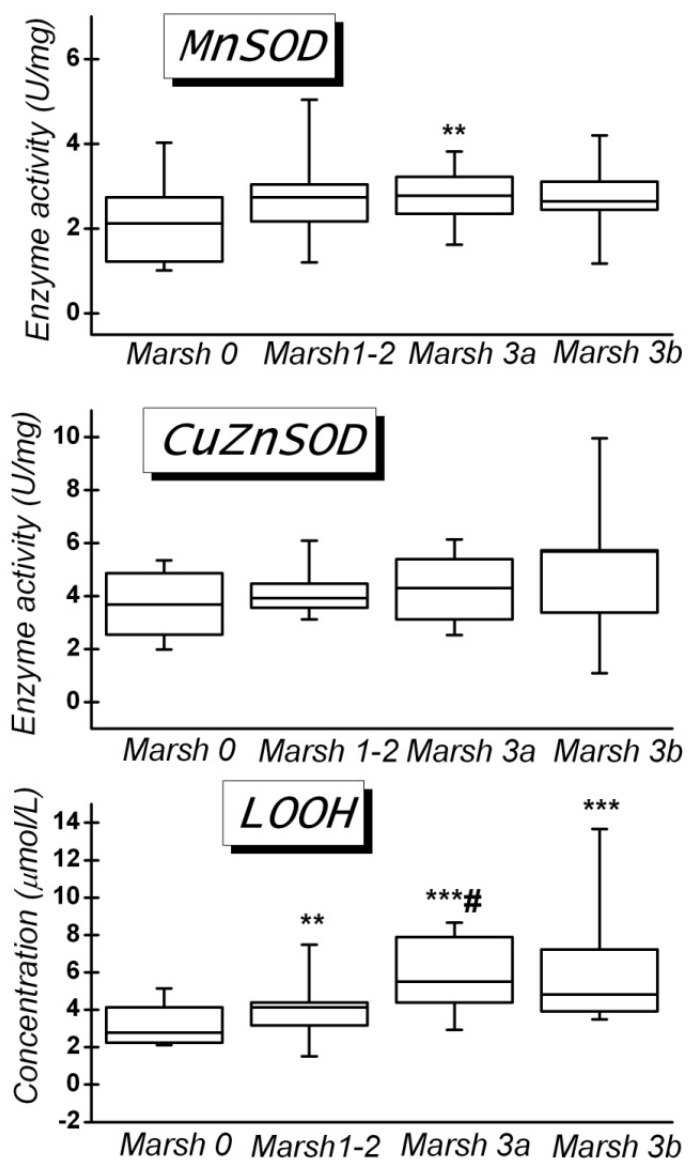

Figure 2. MnSOD andCuZnSOD activity (U/gHb) and LOOH concentration $(\mu \mathrm{mol} / \mathrm{L})$ in the intestinal mucosa of celiac disease patients. ${ }^{* * *} \mathrm{P}<0.001,{ }^{* *} \mathrm{P}<0.01$, significantly different from Marsh o group. \# $\mathrm{P}<0.05$, significantly different from Marsh 1+2 group

The activities of both SODs, as well as the concentration of $\mathrm{LOOH}$ in the intestinal mucosa are represented in Fig. 2. MnSOD activity and $\mathrm{LOOH}$ concentration showed significant differences between the groups: MnSOD: $\mathrm{H}=8.79, P<0.05$; LOOH: $\mathrm{H}=22.92, P<0.001$, while no significant difference between the patient groups was found for CuZnSOD activity $(\mathrm{H}=5.23, P>0.05)$. In comparison to the patients with normal mucosa, MnSOD activity was significantly elevated in Marsh 3a group $(P<0.01)$. Significant increase in $\mathrm{LOOH}$ concentration was found in both groups with villous atrophy (Marsh 3a: $P<0.001$; Marsh 3b: $P<0.01$ ), comparing to Marsh o. In addition, the patients in Marsh 3a group had higher LOOH concentration than the patients in Marsh $1+2$ group.

Relative MnSOD and CuZnSOD protein level in the peripheral blood and intestinal mucosa did not vary significantly between the analyzed groups (Figs. 3 and 4). 


\section{Marsh 0 Marsh 1 Marsh 2 Marsh 3a Marsh 3b}

Figure 3. Relative CuZnSOD protein level in peripheral blood of celiac disease patients

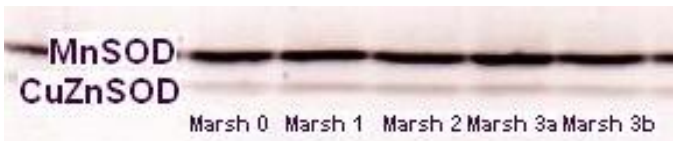

Figure 4. Relative MnSOD and CuZnSOD protein level in the intestinal mucosa of celiac disease patients

Positive correlations were found between the severity of mucosal lesion and CuZnSOD activity ( $\mathrm{r}_{\mathrm{s}}=0.39, P<0.001$ ), as well as $\mathrm{LOOH}$ concentration ( $\mathrm{r}_{\mathrm{s}}=0.52, P<0.001$ ) in the peripheral blood (Fig. 5). Similar correlations were found also in intestinal mucosa: MnSOD: $\mathrm{r}_{\mathrm{s}}=0.27, P<0.05$; CuZnSOD: $\mathrm{r}_{\mathrm{s}}=0.28, P<0.05 ; \mathrm{LOOH}: \mathrm{r}_{\mathrm{s}}=0.55$, $P<0.001$ (Fig. 6).
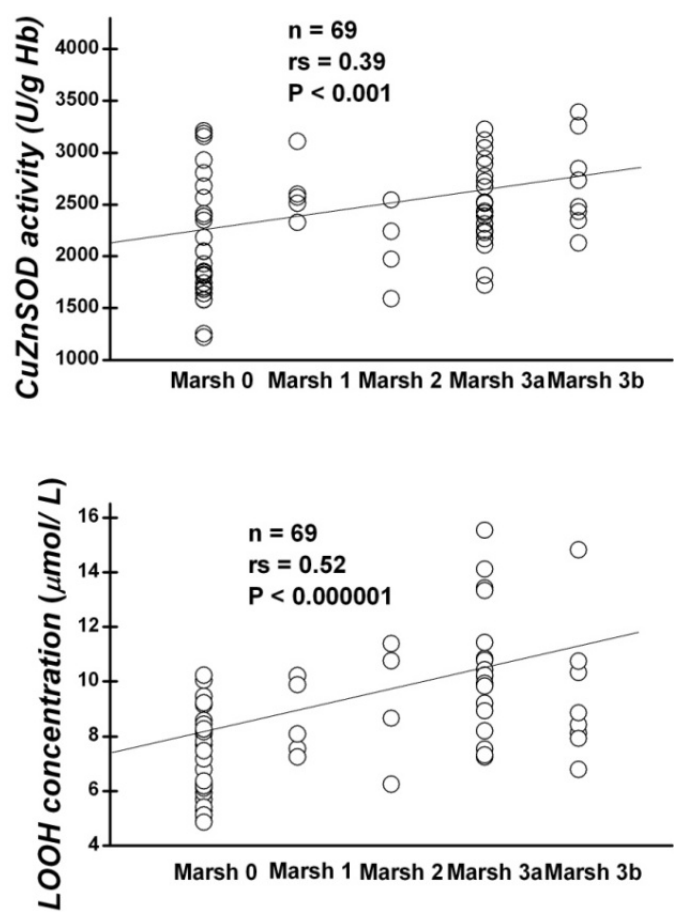

Figure 5. Data plot and coefficients of Spearman's correlation $\mathrm{r}_{\mathrm{s}}$ between CuZnSOD activity and LOOH concentration and the severity of mucosal lesion in the peripheral blood of pediatric patients with celiac disease.
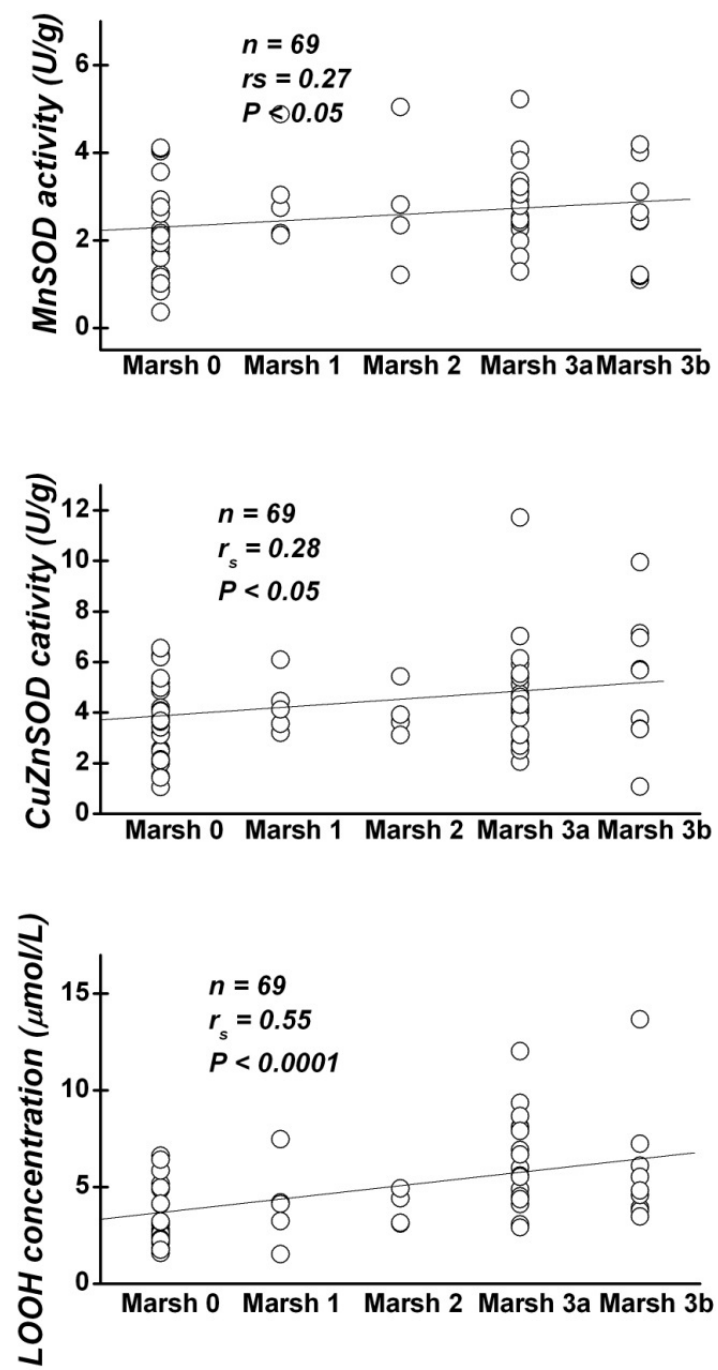

Figure 6. Data plot and coefficients of Spearman's correlation $\mathrm{r}_{\mathrm{s}}$ between MnSOD and CuZnSOD activity and $\mathrm{LOOH}$ concentration and the severity of mucosal lesion in the intestinal mucosa of pediatric patients with celiac disease.

\section{DisCUSSION}

It is well known that free radicals and other ROS are constantly produced in all cells as the by-products of normal cellular metabolism and may play an important role in the pathogenesis of various disorders [9]. Deleterious effects of ROS are counteracted by AO defense system. Superoxide anion radical is the product of the one-electron reduction of oxygen $\mathrm{O}_{2}$, which occurs widely in nature [10] and superoxide dismutases that act as its scavengers are considered to be the first line of AO defense. 
In our study CuZnSOD activity was increased in the blood of patients with partial or subtotal villous atrophy, while SOD protein levels remained stable in comparison to subjects with healthy mucosa. The level of $\mathrm{LOOH}$ was also elevated in the blood of the same patient groups. The increase in the SOD activity probably represents a physiological response to the increased pro-oxidant pressure in $\mathrm{CD}$ patients. Namely, previous reports have shown that, in CD patients, gluten activates pro-oxidant processes that result in an overproduction of free radicals [11]. In spite of higher SOD activity, the elevated plasma concentrations of $\mathrm{LOOH}$ (over 30\%) found in our study, indicate that $\mathrm{AO}$ protection is not adequate in patients with more pronounced mucosal lesions.

Similar alterations were also found in the intestinal mucosa. Both SOD activities were increased in the patients with villous atrophy, although the increase was significant only for MnSOD. These changes of SOD activities, as a response to the increased rate of ROS production, were not caused by the changes in relative protein levels of these enzymes, which were found to be similar in all patient groups. Most probably, the elevation of the SOD activities is a consequence of the posttranslational chemical modification of these enzymes under the changed redox environment [12, 13].

Besides the increased SOD activities, LOOH concentrations were also elevated in the patients with partial or subtotal villous atrophy, not only comparing to the patients with healthy mucosa, but also to those with milder mucosal lesion. The intestinal mucosa is continuously exposed to numerous oxidants, mutagens and carcinogens from diet, as well as to endogenous ROS. In the healthy mucosa, several defense mechanisms are employed to preserve the cell integrity and tissue homeostasis: the intestine is able to maintain high concentrations of antioxidants and to up-regulate antioxidant enzymes, while apoptosis is induced in spent or damaged enterocytes [14]. Our data show that in untreated $\mathrm{CD}$ patients $\mathrm{AO}$ defense system is not capable of maintaining the redox balance, which results in increased lipid peroxidatin and probably oxidative damage of other biomolecules. Elevated LOOH may contribute to the disruption of intestinal detoxifying pathways and to disfunction of enterocytes, leading to various disorders of digestive tract. Our findings are in accordance with the results of Rivabene et al. [15] who found elevated $\mathrm{LOOH}$ in the cell culture after treating the cells with gliadin peptides. Important results of our study are strong correlations between the analyzed parameters and the histological findings. Namely, both SOD activities, as well as LOOH levels significantly positively correlated with the severity of mucosal lesion, pointing to an important role of the oxidative stress and $\mathrm{AO}$ defense system in the pathogenesis of $\mathrm{CD}$. We suggest that $\mathrm{AO}$ parameters should be involved in the analysis, prediction and follow-up of histological changes in patients affected by CD.

Acknowledgement: This work received the financial support of the Ministry of Education, Science and Technological Development of the Republic of Serbia (Grants III 41027 and OI 173041).

\section{REFERENCES}

1. C. Catassi, E. Fabiani, "The spectrum of coeliac disease in children," Bailliere's Clin. Gastroenterol., vol. 11, no. 3, pp. 485-507, Sep. 1997.

DOI: 10.1016/So950-3528(97)90028-2

2. R. J. Farrell, C. P. Kelly, "Celiac sprue,” N. Engl. J. Med., vol. 346, no. 3, pp. 180-188, Jan. 2002.

DOI: 10.1056/NEJMrao10852 PMid: 11796853

3. B. Dugas, P. Debre, S. Moncada, "Nitric oxide, a vital poison inside the immune and inflammatory network," Res. Immunol, vol. 146, no. 9, pp. 664-670, Nov-Dec. 1995 . DOI: 10.1016/0923-2494(96)84914-5

4. P. Odetti et al., "Oxidative stress in subjects affected by celiac disease," Free Radic. Res., vol. 29, no. 1, pp. 17-24, Jul. 1998.

DOI: $10.1080 / 10715769800300031$

PMid: 9733018

5. V. Stojiljkovic et al., "Antioxidant enzymes, glutathione and lipid peroxidation in peripheral blood of children affected by coeliac disease," Ann. Clin. Biochem., vol. 44, no. 6, pp. 537-543, Nov. 2007. Retrieved from:

http://journals.sagepub.com/doi/pdf/10.1258/0004563 07782268075

Retrieved on: Dec. 15, 2016

6. V. Stojiljkovic et al., "Antioxidant status and lipid peroxidation in small intestinal mucosa of children with celiac disease," Clin. Biochem., vol. 42, no. 13-14, pp. 1431-1437, Sep. 2009.

DOI: 10.1016/j.clinbiochem.2009.06.009

PMid: 19560448

7. J. A. Walker-Smith, "Revised criteria for diagnosis of coeliac disease, Report of Working Group of European Society of Paediatric Gastroenterology and Nutrition," Arch. Dis. Child., vol. 65, no. 8, pp. 909-911, Aug. 1990. DOI: 10.1136/adc.65.8.909

8. G. Oberhuber, G. Granditsch, H. Vogelsang, "The histopathology of coeliac disease: time for a standardized report scheme for pathologists," Eur. J. Gastroenterol. Hepatol., vol. 11, no. 10, pp. 1185-1194, Oct. 1999.

DOI: 10.1097/00042737-199910000-00019 PMid: 10524652

9. M. Valko et al., "Free radicals and antioxidants in normal physiological functions and human disease," Int. J. Biochem. Cell Biol., vol. 39, no. 1, pp. 44-84, 2007.

DOI: 10.1016/j.biocel.2006.07.001 PMid: 16978905

10. D. T. Sawyer, "Superoxide Chemistry," AccessScience, 2014. DOI: $10.1036 / 1097-8542.669650$

11. M. Boda, I. Németh and D. Boda, "The caffeine metabolic ratio as an index of xanthine oxidase activity in clinically active and silent celiac patients," J. Pediatr. Gastroenterol. Nutr., vol. 29, no. 5, pp. 546-550, Nov. 1999.

DOI: 10.1097/00005176-199911000-00014 PMid: 10554121

12. F. Yamakura and H. Kawasaki, "Post-translational modifications of superoxide dismutase," Biochim. Biophys. Acta., vol. 1804, no. 2, pp. 318-325, Feb. 2010. DOI: 10.1016/j.bbapap.2009.10.010 PMid: 19837190

13. Y. Furukawa, A. S. Torres, T. V. O'Halloran, "Oxygeninduced maturation of SOD1: a key role for disulfide formation by the copper chaperone CCS," $E M B O J$., vol. 23, no. 14, pp. 2872-2881, Jul. 2004.

DOI: $10.1038 /$ sj.emboj. 7600276

PMid: 15215895 PMCid: PMC1150991

14. T. Y. Aw, "Molecular and cellular responses to oxidative stress and changes in oxidation-reduction imbalance in the intestine," Am. J. Clin. Nutr., vol. 70, no. 4, pp. 557565 , Oct. 1999. 
Retrieved from:

http://ajcn.nutrition.org/content/70/4/557.full.pdf+ht

$\underline{\mathrm{ml}}$

Retrieved on: Dec. 11, 2016

15. R. Rivabene, E. Mancini, M. De Vincenzi, "In vitro cytotoxic effect of wheat gliadin-derived peptides on the Caco-2 intestinal cell line is associated with intracellular oxidative imbalance: implications for coeliac disease," Biochim. Biophys. Acta, vol. 1453, no. 1, pp. 152-16o, Jan. 1999.

DOI: 10.1016/So925-4439(98)00095-7 